\title{
turboTDDFT - A code for the simulation of molecular spectra using the Liouville-Lanczos approach to time-dependent density-functional perturbation theory
}

\author{
Osman Barış Malcıoğlu ${ }^{\mathrm{a}, 1}$, Ralph Gebauer ${ }^{\mathrm{b}, \mathrm{c}, *}$, Dario Rocca ${ }^{\mathrm{d}}$, Stefano Baroni ${ }^{\mathrm{a}, \mathrm{c}}$ \\ a SISSA - Scuola Internazionale Superiore di Studi Avanzati, Trieste, Italy \\ b ICTP - The Abdus Salam International Centre for Theoretical Physics, Trieste, Italy \\ c CNR-IOM DEMOCRITOS Simulation Center, Trieste, Italy \\ d Department of Chemistry, University of California at Davis, Davis, CA, USA
}

\section{A R T I C L E I N F O}

\section{Article history:}

Received 21 December 2010

Received in revised form 6 April 2011

Accepted 12 April 2011

Available online 29 April 2011

\section{Keywords:}

Time-dependent density-functional theory

Density-functional perturbation theory

Quantum ESPRESSO

Optical spectra

Linear response

Liouville equation

Lanczos method

\begin{abstract}
A B S T R A C T
We introduce turboTDDFT, an implementation of the Liouville-Lanczos approach to linearized timedependent density-functional theory, designed to simulate the optical spectra of molecular systems made of up to several hundred atoms. turboTDDFT is open-source software distributed under the terms of the GPL as a component of QUANTUM ESPRESSO. As with other components, turboTDDFT is optimized to run on a variety of different platforms, from laptops to massively parallel architectures, using native mathematical libraries (LAPACK and FFTW) and a hierarchy of custom parallelization layers built on top of MPI.
\end{abstract}

\section{Program summary}

Program title: turboTDDFT

Catalogue identifier: AEIX_v1_0

Program summary URL: http://cpc.cs.qub.ac.uk/summaries/AEIX_v1_0.html

Program obtainable from: CPC Program Library, Queen's University, Belfast, N. Ireland

Licensing provisions: GNU General Public License V 2.0

No. of lines in distributed program, including test data, etc.: 3559889

No. of bytes in distributed program, including test data, etc.: 254283981

Distribution format: tar.gz

Programming language: Fortran 95

Computer: Any computer architecture

Operating system: GNU/Linux, AIX, IRIX, Mac OS X, and other UNIX-like OS's

Classification: $16.2,16.6,7.7$

External routines: turboTDDFT is a tightly integrated component of the Quantum ESPRESSO distribution and requires the standard libraries linked by it: BLAS, LAPACK, FFTW, MPI.

Nature of problem: Calculation of the optical absorption spectra of molecular systems.

Solution method: The dynamical polarizability of a system is expressed in terms of the resolvent of its Liouvillian super-operator within time-dependent density-functional theory, and calculated using a nonHermitean Lanczos method, whose implementation does not require the calculation of any virtual states. Pseudopotentials (both norm-conserving and ultrasoft) are used in conjunction with plane-wave basis sets.

Restrictions: Spin-restricted formalism. Linear-response regime. No hybrid functionals. Adiabatic XC kernels only.

Unusual features: No virtual orbitals are used, nor even calculated. A single Lanczos recursion gives access to the whole optical spectrum.

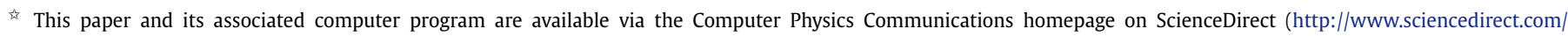
science/journal/00104655).

* Corresponding author at: ICTP - The Abdus Salam International Centre for Theoretical Physics, Trieste, Italy.

E-mail address: rgebauer@ictp.it (R. Gebauer).

1 Present address: Département de Physique, Université de Liège, B-5, 4000 Sart-Tilman, Belgium.
} 
Additional comments: !!!!! The distribution file for this program is over 254 Mbytes and therefore is not delivered directly when download or E-mail is requested. Instead a html file giving details of how the program can be obtained is sent.

Running time: From a few minutes for small molecules on serial machines up to many hours on multiple processors for complex nanosystems with hundreds of atoms.

(C) 2011 Elsevier B.V. All rights reserved.

\section{Introduction}

Time-dependent density-functional theory (DFT [1,2], TDDFT [3, $4]$ ) is gaining increasing popularity as a powerful method for the numerical simulation of optical spectra in a variety of molecular systems. In spite of its numerous successes, TDDFT is still affected by two major limitations. The first, and more fundamental, is the inability of static, local, exchange-correlation (XC) kernels to represent the long-range tail of the electron-hole interaction, which is essential to account for charge-transfer excitations, as well as excitons in solids and Rydberg series in molecules [5-7]. The second, more practical, difficulty stems from the poor capacity of current implementations of TDDFT to address extended portions of the spectrum of complex molecular systems (made of several hundreds of independent atoms) [8,9]. The present paper addresses the second difficulty by making a new, and supposedly superior, implementation of (linearized) TDDFT [8-10] widely available to the scientific community.

Current implementations of TDDFT fall into three broad categories. In the first, the TDDFT charge susceptibility is obtained from the independent-electron susceptibility using a Dyson-like linear equation [12,13]; in the second, the poles of the susceptibility, corresponding to excitations energies, are addressed as the eigenvalues of a suitable linear (super-) operator equation [13,14]; finally, the full spectrum of a system can be obtained by Fourier analyzing the time series generated by the expectation value of some observable (such as the e.g. dipole) calculated along the perturbed time evolution of the TDDFT molecular orbitals $[15,16]$. The relative merits of each one of these approaches are discussed in Ref. [9], where an alternative approach to the calculation of optical spectra within TDDFT, named the Liouville-Lanczos method, is also presented, following a suggestion originally proposed in Ref. [8]. The distinctive feature of the new method is that it allows for the full spectrum of a system to be calculated over a broad frequency range, with a computational effort which is only a few times larger than that needed by a single ground-state DFT or static densityfunctional perturbation theory (DFPT) calculation [17,18].

In this paper we introduce a computer code, named turboTDDFT, which implements the Liouville-Lanczos approach to TDDFT, and which is being distributed under the terms of the GPL license [19], as a component of the QUANTUM ESPRESSO suite of open-source DFT plane-wave (PW) pseudopotential codes [20-22]. In Section 2 we provide a minimal theoretical background and establish some notation for the problem addressed by turboTDDFT; in Section 3 we describe the algorithm implemented in turboTDDFT; in Section 4 we describe turboTDDFT as a component of the QUANTUM ESPRESSO distribution; in Section 5 we provide the instructions for installing turboTDDFT on UNIX systems; in Section 6 we give a few examples of the usage of turboTDDFT for the calculation of the spectra of some prototypical simple molecular systems; Section 7 finally contains our conclusions and perspectives for future work.

\section{Statement of the problem, minimal theoretical background, and notation}

In the dipole approximation, the response of molecular systems to electromagnetic radiation is described by the dynamical polarizability tensor, $\alpha_{i j}(\omega)$, whose elements are defined as the dipole moment linearly induced along the $i$-th Cartesian direction by a perturbing electric field of unit strength, polarized along the $j$-th axis, and oscillating at the frequency $\omega$. The absorption coefficient is essentially the product of the frequency times the imaginary part of the diagonal elements (or trace) of the polarizability [23]. In this paper we address the dynamical polarizability of a molecular system at clamped nuclei, and we use atomic (Hartree) units throughout: $\hbar=1 ; e=1 ; m_{e}=1$. Since the current implementation of turboTDDFT treats spin-restricted systems only, we exclude in the following spin degrees of freedom from our notations for simplicity.

The polarizability of a system of interacting electrons can be expressed as:

$\alpha_{i j}(\omega)=\operatorname{Tr}\left(\hat{X}_{i} \hat{\rho}_{j}^{\prime}(\omega)\right)$,

where carets indicate quantum mechanical operators, $\hat{X}_{i}$ is the $i$-th component of the dipole (or position) operator, $\hat{\rho}_{j}^{\prime}(\omega)=\hat{\rho}_{j}(\omega)-$ $\hat{\rho}^{\circ}$, is the response density matrix, $\hat{\rho}_{j}(\omega)$ being the one-electron density matrix of the system perturbed by an external homogeneous electric field of unit strength polarized along the $j$-th Cartesian axis and oscillating at frequency $\omega$, and $\hat{\rho}^{\circ}$ is its unperturbed counterpart. In TDDFT the response density matrix can be expressed as the solution of the linearized quantum Liouville equation [8-11]:

$(\omega-\mathcal{L}) \cdot \hat{\rho}_{j}^{\prime}(\omega)=\left[\hat{X}_{j}, \hat{\rho}^{\circ}\right]$,

where $\mathcal{L}$ is the TDDFT Liouvillian of the system, defined as:

$\mathcal{L} \cdot \hat{\rho}^{\prime} \triangleq\left[\hat{H}^{\circ}, \hat{\rho}^{\prime}\right]+\left[\hat{V}_{H X C}^{\prime}\left[\hat{\rho}^{\prime}\right], \hat{\rho}^{\circ}\right]$,

$\hat{H}^{\circ}$ is the unperturbed Kohn-Sham (KS) Hamiltonian [2], $\hat{V}_{H X C}^{\prime}\left[\hat{\rho}^{\prime}\right]$ is the linear correction to the Hartree-plus-XC potential, whose coordinate representation is:

$v_{H X C}^{\prime}(\mathbf{r}, \omega)=\int\left(\frac{1}{\left|\mathbf{r}-\mathbf{r}^{\prime}\right|}+\kappa_{X C}\left(\mathbf{r}, \mathbf{r}^{\prime} ; \omega\right)\right) \rho^{\prime}\left(\mathbf{r}^{\prime}, \mathbf{r}^{\prime} ; \omega\right) d \mathbf{r}^{\prime}$,

and $\kappa_{X C}$ is the so-called XC kernel [12] that, in the adiabatic DFT approximation [24], is independent of $\omega$. Traces of products of operators, such as in Eq. (1), have the same algebra as scalar products in linear spaces, $\operatorname{Tr}\left(\hat{A}^{\dagger} B\right) \triangleq(\hat{A}, \hat{B})$, and this property is instrumental in expressing the polarizability as an off-diagonal matrix element of the resolvent of the Liouvillian [8,9]. By solving the linear equation (2) we can express the polarizability in Eq. (1) as

$\alpha_{i j}(\omega)=-\left(\hat{X}_{i},(\omega-\mathcal{L})^{-1} \cdot\left[\hat{X}_{j}, \hat{\rho}^{\circ}\right]\right)$,

where $(\bullet, \bullet)$ indicates the scalar product between two operators, in the sense defined above. Of course, in order to give a well-defined meaning to Eq. (5), a well-defined representation must be given for 
operators, super-operators (i.e. operators acting on the linear space of quantum mechanical operators), and for scalar products defined in this linear space. This will be done in Section 2.1.

The coordinate representation of the response density matrix is:

$\rho^{\prime}\left(\mathbf{r}, \mathbf{r}^{\prime}, \omega\right)=2 \sum_{v=1}^{N_{v}}\left(\tilde{\varphi}_{v}^{\prime}(\mathbf{r}, \omega) \varphi_{v}^{\circ *}\left(\mathbf{r}^{\prime}\right)+\varphi_{v}^{\circ}(\mathbf{r}) \tilde{\varphi}_{v}^{\prime *}\left(\mathbf{r}^{\prime},-\omega\right)\right)$,

where $\varphi_{v}^{\circ}(\mathbf{r})$ are unperturbed KS orbitals, $\tilde{\varphi}_{v}^{\prime}(\mathbf{r}, \omega)=\varphi_{v}(\mathbf{r}, \omega)-$ $\varphi_{v}^{\circ}(\mathbf{r})$ denotes the first-order correction to the $v$-th KS orbital, and $N_{v}$ is the number of occupied KS states; the factor 2 accounts for the spin degeneracy of molecular orbitals in non-magnetic systems. Note that in the frequency domain the density matrix is not Hermitian. Being the Fourier transform of a Hermitian operator, it satisfies the relation: $\hat{\rho}(\omega)^{\dagger}=\hat{\rho}(-\omega)$.

\subsection{Representation of the response density matrix and of other operators}

As shown in Eq. (6), the response density matrix at any given frequency $\omega$ is uniquely determined by the two sets of response orbitals $\left\{\tilde{\varphi}_{v}^{\prime}(\mathbf{r}, \omega)\right\}$ and $\left\{\tilde{\varphi}_{v}^{\prime}(\mathbf{r},-\omega)\right\}$. Standard time-dependent perturbation theory indicates that each response orbital $\tilde{\varphi}_{v}^{\prime}$ can be chosen to be orthogonal to the KS occupied-state manifold. For this reason the response density matrix of Eq. (6) has vanishing matrix elements between pairs of occupied and unoccupied states, namely $\left\langle\varphi_{c}^{\circ}\left|\hat{\rho}^{\prime}\right| \varphi_{c^{\prime}}^{\circ}\right\rangle=\left\langle\varphi_{v}^{\circ}\left|\hat{\rho}^{\prime}\right| \varphi_{v^{\prime}}^{\circ}\right\rangle=0 \forall\left(v, v^{\prime}\right)$ and $\left(c, c^{\prime}\right)$, where $v$ and $v^{\prime}$ denotes generic occupied (valence) states and $c$ and $c^{\prime}$ generic empty (conduction) states. This is to say that in the representation of the unperturbed KS states the response density matrix has the block structure:

$\hat{\rho}^{\prime} \rightarrow\left(\begin{array}{cc}0 & \rho_{v c}^{\prime} \\ \rho_{c v}^{\prime} & 0\end{array}\right)$

This block structure, which also holds for the commutator $\left[\hat{X}_{j}, \hat{\rho}^{\circ}\right]$, allows us to conveniently represent the operators appearing in Eqs. (3)-(5) using sets of $2 N_{v}$ orbitals, rather than square matrices. Using such a representation would require the storage of $n=2 N N_{v}$ coefficients to represent the orbitals, $N$ being the dimension of the one-electron basis set, which is clearly much more convenient than storing and using the $N^{2}$ entries of the full representation of the density matrix. Inspired by these considerations we define the standard batch representation (SBR) of the response density matrix as:

$\hat{\rho}^{\prime} \stackrel{S B R}{\longrightarrow}\left(\begin{array}{l}\left\{q_{v}\right\} \\ \left\{p_{v}\right\}\end{array}\right)$,

where $\left\{q_{v}\right\}$ and $\left\{p_{v}\right\}$ indicate the sets (batches) of orbitals:

$q_{v}(\mathbf{r})=\frac{1}{2}\left(\tilde{\varphi}_{v}^{\prime}(\mathbf{r}, \omega)+\tilde{\varphi}_{v}^{\prime *}(\mathbf{r},-\omega)\right)$,

$p_{v}(\mathbf{r})=\frac{1}{2}\left(\tilde{\varphi}_{v}^{\prime}(\mathbf{r}, \omega)-\tilde{\varphi}_{v}^{\prime *}(\mathbf{r},-\omega)\right)$

Note that in a time-reversal invariant system the response charge density can be expressed in terms of the $\left\{q_{v}\right\}$ orbitals alone:

$n^{\prime}(\mathbf{r}, \omega)=4 \sum_{v} \varphi_{v}^{\circ}(\mathbf{r}) q_{v}(\mathbf{r})$

General one-particle quantum mechanical operators can be given a similar representation. The SBR of a general operator is defined as:

$\hat{A} \stackrel{S B R}{\longrightarrow}\left(\begin{array}{l}\left\{a_{v}^{q}\right\} \\ \left\{a_{v}^{p}\right\}\end{array}\right) \triangleq a$, where the orbitals $a_{v}^{q}(\mathbf{r})$ and $a_{v}^{p}(\mathbf{r})$ are defined as

$a_{v}^{q}(\mathbf{r})=\frac{1}{2}\left(\hat{Q} \hat{A} \varphi_{v}^{\circ}(\mathbf{r})+\left(\hat{Q} \hat{A}^{\dagger} \varphi_{v}^{\circ}(\mathbf{r})\right)^{*}\right)$,

$a_{v}^{p}(\mathbf{r})=\frac{1}{2}\left(\hat{Q} \hat{A} \varphi_{v}^{\circ}(\mathbf{r})-\left(\hat{Q} \hat{A}^{\dagger} \varphi_{v}^{\circ}(\mathbf{r})\right)^{*}\right)$,

$\hat{Q}$ is the projector over the KS empty-state manifold. In our implementation we avoid the calculation of empty states by using the relation: $\hat{Q}=\hat{I}-\hat{P}$, where $\hat{I}$ is the identity operator and $\hat{P}=\sum_{v=1}^{N_{v}}\left|\varphi_{v}^{\circ}\right\rangle\left\langle\varphi_{v}^{\circ}\right|$ is the projector onto the occupied-state manifold; this definition of $\hat{Q}$ clearly requires only the occupied states $\varphi_{v}^{\circ}$ [17]. The $\left\{a_{v}^{q}\right\}$ and $\left\{a_{v}^{p}\right\}$ functions of Eqs. (13)-(14) will be referred to as the upper (or $q$-like) and lower (or $p$-like) components of the SBR of the $\hat{A}$ operator. If $\hat{A}$ is a Hermitian operator, then its SBR is given by

$\hat{A}=\hat{A}^{\dagger} \stackrel{S B R}{\longrightarrow}\left(\begin{array}{c}\left\{\hat{Q} \hat{A} \varphi_{v}^{\circ}\right\} \\ \{0\}\end{array}\right)$,

where we have assumed that the ground-state orbitals $\varphi_{v}^{\circ}$ are real, as they can always be chosen for time-reversal invariant problems. Other operators appearing in Eq. (2) are represented as:

$\left[\hat{H}^{\circ}, \hat{\rho}^{\prime}\right] \stackrel{S B R}{\longrightarrow}\left(\begin{array}{c}\left\{\left(\hat{H}^{\circ}-\varepsilon_{v}^{\circ}\right) p_{v}\right\} \\ \left\{\left(\hat{H}^{\circ}-\varepsilon_{v}^{\circ}\right) q_{v}\right\}\end{array}\right)$,

$\left[\hat{A}, \hat{\rho}^{\circ}\right] \stackrel{S B R}{\longrightarrow}\left(\begin{array}{c}\{0\} \\ \left\{\hat{Q} \hat{A} \varphi_{v}^{\circ}\right\}\end{array}\right)$,

where $\varepsilon_{v}^{\circ}$ are unperturbed KS orbital energies. Clearly the SBR of an operator is incomplete because it misses the information contained in the matrix blocks that vanish in the KS-state representation of the response density matrix, Eq. (8). It is however sufficient to calculate traces of products of any operator with any response density matrix having the block structure of Eq. (7). By using the SBR, the polarizability in Eq. (5) can be expressed as:

$\alpha_{i j}(\omega)=-4\left(x_{i},(\omega-L)^{-1} \cdot y_{j}\right)$

where $x_{i}, y_{j}$, and $L$ are the SBR representations of $\hat{X}_{i},\left[\hat{X}_{j}, \hat{\rho}^{\circ}\right]$, and of the Liouvillian, respectively:

$\hat{X}_{i} \stackrel{S B R}{\longrightarrow}\left(\begin{array}{c}\left\{x_{i, v}\right\} \\ \{0\}\end{array}\right) \triangleq x_{i}$

$\left[\hat{X}_{j}, \hat{\rho}^{\circ}\right] \stackrel{S B R}{\longrightarrow}\left(\begin{array}{c}\{0\} \\ \left\{x_{j, v}\right\}\end{array}\right) \triangleq y_{j}$,

$\mathcal{L} \stackrel{S B R}{\longrightarrow}\left(\begin{array}{cc}0 & D \\ D+2 K & 0\end{array}\right) \triangleq L$,

the $x_{i, v}$ orbitals are defined as

$x_{i, v}(\mathbf{r})=\hat{Q} \hat{X}_{i} \varphi_{v}^{\circ}(\mathbf{r})$,

and the $D$ and $K$ super-operators are defined as:

$D \cdot\left\{u_{v}(\mathbf{r})\right\}=\left\{\left(\hat{H}^{\circ}-\varepsilon_{v}^{\circ}\right) u_{v}(\mathbf{r})\right\}$,

$K \cdot\left\{u_{v}(\mathbf{r})\right\}=\left\{4 \varphi_{v}^{\circ}(\mathbf{r}) \sum_{v^{\prime}} \int \kappa\left(\mathbf{r}, \mathbf{r}^{\prime}\right) \varphi_{v^{\prime}}^{\circ}\left(\mathbf{r}^{\prime}\right) u_{v^{\prime}}\left(\mathbf{r}^{\prime}\right) d \mathbf{r}^{\prime}\right\}$.

Finally, the SBR of scalar products (traces of products of operators) reads:

$\operatorname{Tr}\left(\hat{A}^{\dagger} \hat{B}\right) \stackrel{S B R}{\longrightarrow} \sum_{v=1}^{N_{v}}\left(\left\langle a_{v}^{q} \mid b_{v}^{q}\right\rangle+\left\langle a_{v}^{p} \mid b_{v}^{p}\right\rangle\right) \triangleq(a, b)$,

where $\left(\left\{a_{v}^{q},\right\}\left\{a_{v}^{p}\right\}\right),\left(\left\{b_{v}^{q},\right\}\left\{b_{v}^{p}\right\}\right)$ are the SBRs of $\hat{A}$ and $\hat{B}$, respectively, and brackets $\langle\bullet \mid \bullet\rangle$ indicate standard quantum-mechanical 
scalar products between one-electron orbitals. Note that, according to these definitions, the two operators in Eq. (5), as well as their SBR in (18), are orthogonal because the commutator of two Hermitian operators is anti-Hermitian and the trace of the product of a Hermitian and an anti-Hermitian operator vanishes.

\subsection{Dipole operator in periodic boundary conditions}

In order to obtain the SBR of the dipole operator and of its commutator with the unperturbed density matrix, Eqs. (19)-(20), one needs to evaluate the orbitals defined in Eq. (22). In periodic boundary conditions (such as used in our PW implementation) the position operator $\hat{X}_{i}$ is ill defined, since it is both non-periodic and not bound from below. As a consequence it is not possible to compute the expectation value of $\hat{X}_{i}$ on Bloch states. However in the calculation of $\hat{Q} \hat{X}_{i} \varphi_{v}^{\circ}$ only off-diagonal matrix elements of $\hat{X}_{i}$ are required:

$$
\hat{Q} \hat{X}_{i} \varphi_{v}^{\circ}=\sum_{c}\left|\varphi_{c}^{\circ}\right\rangle\left\langle\varphi_{c}^{\circ}\left|\hat{X}_{i}\right| \varphi_{v}^{\circ}\right\rangle
$$

which are well defined in periodic boundary conditions [28]. Indeed, one has

$\left\langle\varphi_{c}^{\circ}\left|\hat{X}_{i}\right| \varphi_{v}^{\circ}\right\rangle=\frac{1}{\left(\varepsilon_{c}^{\circ}-\varepsilon_{v}^{\circ}\right)}\left\langle\varphi_{c}^{\circ}\left|\left[\hat{H}^{\circ}, \hat{X}_{i}\right]\right| \varphi_{v}^{\circ}\right\rangle$,

and, if the potential operator in the unperturbed Hamiltonian is purely local, then the commutator in Eq. (27) is simply proportional to the momentum operator,

$\left[\hat{H}^{\circ}, \hat{X}_{i}\right]=-\frac{\hbar^{2}}{m} \hat{P}_{i}$

When the potential acting on electrons has non-local contributions (which is the case for the vast majority of pseudopotentials), an explicit correction due to those non-local terms must be added to the momentum operator in Eq. (28) [29,30].

In practice, turboTDDFT is designed so as to avoid any explicit reference to virtual eigenpairs of the KS Hamiltonian, so that Eq. (27) cannot be used directly. However the relevant $\hat{Q} \hat{X}_{i} \varphi_{v}^{\circ}$ orbitals can be obtained directly by solving a set of linear systems, as proposed in Ref. [17] and thoroughly explained in Ref. [18]. The relevant routines used to accomplish this task are borrowed from the phonon code in the QUANTUM ESPRESSO distribution [21].

\section{Algorithm}

According to the discussion in the previous section, any component of the polarizability tensor can be expressed as an offdiagonal element of the resolvent of the Liouvillian (super-) operator. Let us now see how such matrix elements can be conveniently calculated using a generalization of the recursion method by Haydock, Heine, and Kelly [31].

\subsection{Calculation of the polarizability}

At first sight, it may seem that the calculation of the polarizability from the resolvent of the Liouvillian, Eq. (18), would require the solution of an $n \times n$ linear system to invert $(\omega-L)$ for each different value of the frequency $\omega$, a very demanding task as the system size and/or the number of frequencies increase. As an expedient alternative, turboTDDFT uses a specially tailored iterative method, called the Lanczos bi-orthogonalization algorithm (LBOA) [9, $32,33]$, which allows for the bulk of the numerical work to be done once for all the frequencies, while the linear system is inexpensively solved in an approximate representation where the matrix to be inverted is both tridiagonal and of much smaller size $[8,9]$. By starting from the initial pair of vectors $u^{1}=v^{1}=y_{j}$, this algorithm computes two coupled Lanczos chains, generated by recursively applying $L$ and $L^{\top}$ to the previous chain vectors. A pair of bi-orthogonal basis sets of increasing dimension $m$ are thus recursively constructed, such that the following factorization holds:

$L \cdot{ }^{m} V \approx{ }^{m} V \cdot{ }^{m} T+\beta^{m+1} v^{m+1 m} e_{m}^{\top}$,

$L^{\top} \cdot{ }^{m} U \approx{ }^{m} U \cdot{ }^{m} T^{\top}+\gamma^{m+1} u^{m+1 m} e_{m}^{\top}$,

${ }^{m} U^{\top} \cdot{ }^{m} V={ }^{m} I$

where ${ }^{m} V=\left[v^{1}, v^{2}, \ldots, v^{m}\right]$ and ${ }^{m} U=\left[u^{1}, u^{2}, \ldots, u^{m}\right]$ are $n \times m$ rectangular matrices whose columns are the elements of the biorthogonal basis sets, $v^{l}$ and $u^{l}$, generated through the LBOA (and referred to as the right and left iterates of the Lanczos recursion), ${ }^{m} T$ is the $m \times m$ tridiagonal matrix made out of the LBOA coefficients,

${ }^{m} T=\left(\begin{array}{ccccc}0 & \gamma^{2} & & & 0 \\ \beta^{2} & 0 & \gamma^{3} & & \\ & \beta^{3} & 0 & \ddots & \\ & & \ddots & \ddots & \gamma^{m} \\ 0 & & & \beta^{m} & 0\end{array}\right)$,

$m e_{l}$ indicates the $l$-th unit vector in an $m$-dimensional space, and ${ }^{m} I$ is the $m \times m$ unit matrix. The diagonal matrix elements in Eq. (32) vanish because of the special structure of the Liouvillian, Eq. (21), and of the starting vector $y_{j}$, Eq. (20); one also has: $\left|\beta^{l}\right|=\left|\gamma^{l}\right|$. Using the factorization of Eq. (29), and neglecting the terms proportional to $\beta^{m+1}$ and $\gamma^{m+1}$ therein, the molecular polarizability given by Eq. (18) can be cast into the form:

$$
\begin{aligned}
\alpha_{i j}(\omega) & \approx 4\left({ }^{m} z_{i j},\left(\omega-{ }^{m} T_{j}\right)^{-1} \cdot{ }^{m} e_{1}\right) \\
& =4\left({ }^{m} z_{i j},{ }^{m} w_{j}(\omega)\right),
\end{aligned}
$$

where ${ }^{m} z_{i j}={ }^{m} V_{j}^{\top} \cdot x_{i}$ is an $m$-dimensional column array whose components can be calculated on the fly at every Lanczos iteration $l$ as

$$
\begin{aligned}
z_{i j}^{l} & =\left(x_{i}, v_{j}^{l}\right) \\
& =\sum_{v=1}^{N_{v}}\left\langle x_{i, v} \mid v_{j, v}^{q, l}\right\rangle,
\end{aligned}
$$

the orbitals $\left\{x_{i, v}\right\}$ are defined in Eq. (22), $v_{j, v}^{q, l}$ is the $v$-th upper ( $q$ like) component of the Lanczos vector $v_{j}^{l}$, and the $m$-dimensional column array ${ }^{m} w_{j}(\omega)$ is solution of the tridiagonal linear system:

$\left(\omega-{ }^{m} T_{j}\right) \cdot{ }^{m} w_{j}(\omega)={ }^{m} e_{1}$.

In the last three equations we have appended a $j$ suffix to the ${ }^{m} T, v$, and ${ }^{m} V$ arrays to indicate that they correspond to different Lanczos chains generated for different polarizations of the perturbing electric field, $X_{j}$.

In practice, the procedure outlined above is performed in two steps, for any given external perturbation (such as, e.g., different polarizations $j$ of the perturbing electric field). The first step, which is also the most time consuming, generates the tridiagonal matrix ${ }^{m} T$ and the ${ }^{m} z$ array, whose components are obtained on the fly at each Lanczos iteration using Eq. (36). The calculation of several response functions (such as, e.g., different components of 


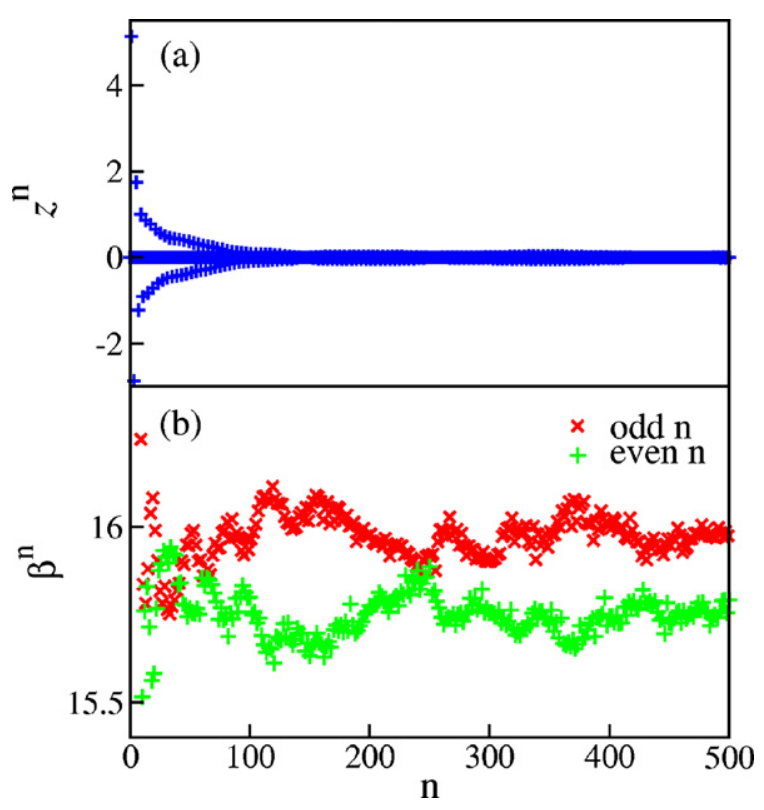

Fig. 1. (a): Numerical behavior of the components of the ${ }^{m} z$ array (Eqs. (35) and (36)) for the fullerene $C_{60}$ molecule; after about 100 iterations the values become quite small. (b): Numerical behavior of the $\beta$ coefficients (Eq. (32)) calculated for the same system, as functions of the iteration count $n$. The values tend to a constant that is approximately half the kinetic-energy cutoff (30 Ry) and odd and even coefficients oscillate with a width $(3.17 \mathrm{eV})$ that is approximately twice the calculated optical gap $(1.6 \mathrm{eV})$. The numerical detail of the simulation are the same as described in Sections 4.1-4.3.

the molecular dipole, corresponding to different rows of the polarizability tensor) implies the simultaneous calculation of different ${ }^{m} z$ arrays. In the second step, the response functions are calculated from Eq. (34) upon solution of Eq. (37), for any different frequency one is interested in. Because of the tridiagonal form and the small dimension of the matrix ${ }^{m} T$, the second step is computationally much less demanding than the first one. In the turboTDDFT package each one of these two steps is implemented in a different executable: the second one will likely be run on desktop or even laptop computers, whereas the first may require substantial computer resources, according to the size of the system. More details on the LBOA as applied to linearized TDDFT can be found in Ref. [9].

\subsection{Extrapolating the Lanczos recursion}

The components of the $\mathrm{m}_{Z}$ array in Eq. (34) decrease rather rapidly when the number of iterations grows large, so that only a relatively small number of components have to be explicitly calculated. A much larger number of $\beta$ and $\gamma$ coefficients is however necessary to have the solution of Eq. (37) converge. In Ref. [9] it was shown that, for large iteration counts, $\beta$ and $\gamma$ oscillate around two distinct values for odd and even iterates, whose average is related to the energy width of the calculated spectrum (which in a PW representation is essentially given by the kineticenergy cutoff), and whose difference is related to the optical gap.

In Fig. 1 we display the typical behavior of the elements of the ${ }^{m} z$ array and of the Lanczos $\beta$ coefficients (see Eq. (32)) as functions of the Lanczos iteration count for the case of the fullerene $\mathrm{C}_{60}$ molecule (see Section 4). Note that for large iteration counts the $\beta$ coefficients oscillate around a value that is roughly half the kinetic-energy cutoff (here: $30 \mathrm{Ry}$ ), and that the difference between the averages for even and odd iteration counts (here: $3.17 \mathrm{eV}$ ) is roughly twice as large as the calculated optical gap, which is $1.6 \mathrm{eV}$ in this system (the gap in the Liouvillian spectrum is also twice as large as the optical gap because the spectrum comprises positive and negative frequencies).

The rapid decrease of the components of the ${ }^{m} z$ array, together with the observed asymptotic behavior of the tridiagonal coefficients suggest an effective strategy to enhance the accuracy of the Liouville-Lanczos algorithm by extrapolating the results obtained from a relatively small number of iterations: once $m$ Lanczos iterations are performed and the regime is attained where further components of the $z$ array are negligible and the $\beta$ and $\gamma$ coefficients display the typical bi-modal behavior of Fig. 1, a (much) larger tridiagonal system is solved, where the missing components of $z$ are simply set to zero, whereas the missing values of $\beta$ and $\gamma$ are set to the average of the values which have been actually calculated. Using distinct averages for odd and even iteration counts may actually result in a slightly improved accuracy of the extrapolation. An example of the efficiency of this procedure will be shown in Section 4.

\subsection{Representation of other response functions}

One of the main advantages of the Liouville-Lanczos approach to TDDFT is that it gives direct access to the observable spectrum without having to calculate (and eventually dispose of) individual eigenvalues of the Liouvillian (which are numerical artifacts of the calculation in the continuum of the spectrum) and its eigenvectors (which contain way more information than accessible in any experiment). One drawback is that it does not allow one to use the orbital-based techniques commonly employed in quantum chemistry to identify the character of individual spectral features [14]. Selection rules may help to some extent, but specific tools giving access to more detailed information about individual spectral lines would be extremely useful. The many different response functions that can be evaluated corresponding to a same perturbation using turboTDDFT constitute a natural toolkit to analyze the features of the calculated spectra. One such response function, which gives visual information on the photo-active regions of complex molecular systems, is the real-space electron charge-density response to a perturbing electric field, $n^{\prime}(\mathbf{r}, \omega)$, given in Eq. (11).

\subsubsection{Charge-density response}

The response charge density can in principle be calculated from Eq. (11), which would however require the preliminary calculation of the (SBR of the) response density matrix. This is impractical for large systems, and a more direct approach can be followed instead. Using arguments similar to those leading to the expression, Eq. (34), for the molecular polarizability, the charge-density response to a homogeneous electric field polarized along the $j$-th direction can be cast into the form:

$n_{j}^{\prime}(\mathbf{r}, \omega)=4\left({ }^{m} z_{j}(\mathbf{r}),{ }^{m} w_{j}(\omega)\right)$,

where the $l$-th component of the ${ }^{m} z_{j}(\mathbf{r})$ array reads:

$m_{j}^{l}(\mathbf{r})=\sum_{v} \varphi_{v}^{\circ}(\mathbf{r}) v_{j, v}^{q, l}(\mathbf{r})$

and $v_{j, v}^{q, l}$ has the same meaning as in Eq. (36).

In PW calculations, real-space quantities - such as chargedensity distributions - are usually represented over 3D grids whose size is a few times larger than the number of PWs and can contain as many as hundreds of thousands points in several challenging applications. A direct evaluation of the response density from Eqs. (38)-(39) would require at each Lanczos step $l$ and for each grid point $\mathbf{r}$ the storage of $z_{j}^{l}(\mathbf{r})$. The huge amount of memory required would make this task impractical in most applications. Therefore, a two-step procedure is used instead. A first Lanczos 

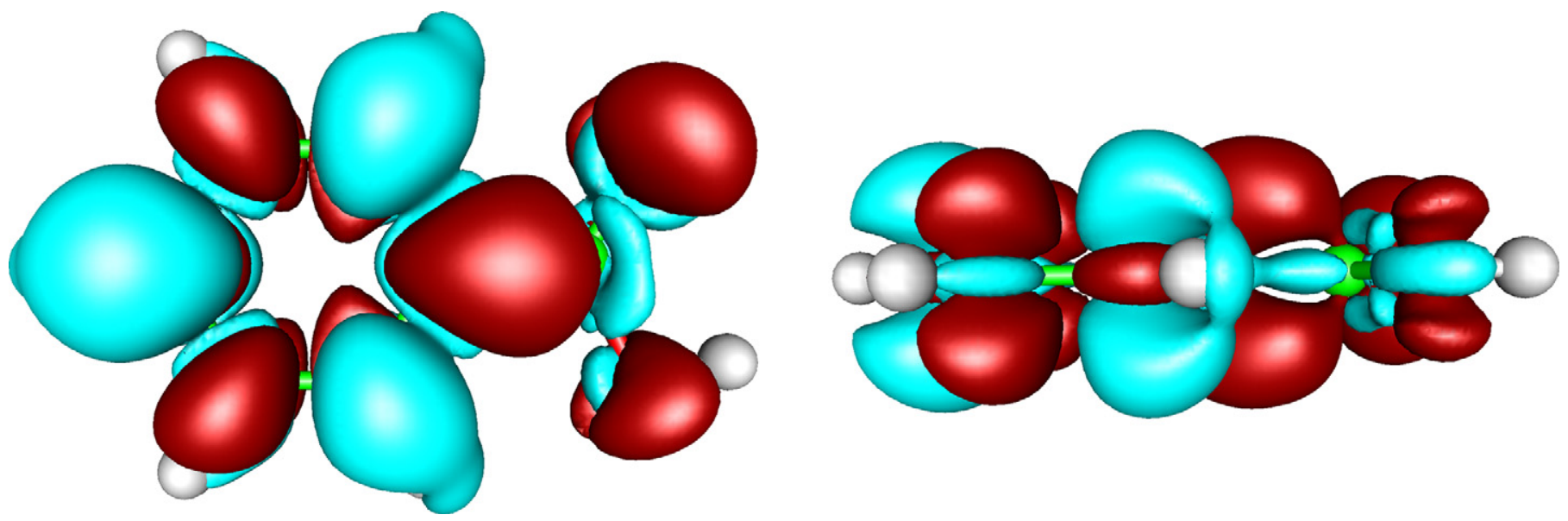

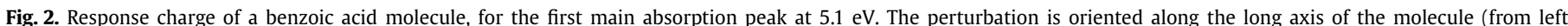
to right in this figure). The two panels show a top view and side view, respectively.

recursion is performed to obtain the recursion coefficients $\beta^{l}, \gamma^{l}$ and the optical spectrum. From the spectrum, the frequencies $\omega$ of interest can be determined and using the $\beta^{l}, \gamma^{l}$ coefficients, the ${ }^{m} w_{j}(\omega)$ array is calculated for those frequencies of interest, using Eq. (37).

In order to determine the response charge density at those frequencies, a second Lanczos recursion chain is performed and since now the ${ }^{m} w_{j}(\omega)$ are known from the previous step, the contribution of each iteration can be summed to evaluate Eq. (38), without storing the $z_{j}$ coefficients of the previous iterations. In this way, the problem of excessive memory use is avoided, albeit at the price of a second Lanczos recursion chain.

In Fig. 2 we illustrate the concept of response charges with the simple example of a benzoic acid molecule. The response charge has been computed for the first main absorption peak (at $5.1 \mathrm{eV}$ ), for an electric field polarized along the long axis of the molecule.

\section{Description and use of individual software components}

The turboTDDFT code is designed as a module for the QUANTUM ESPRESSO distribution. It resides in a self contained directory under the root directory of the QUANTUM ESPRESSO tree. When compiled (see Section 5), the bin/ directory in the QUANTUM ESPRESSO root contains links to the executables turbo_ lanczos.x (the main program) and turbo_spectrum.x (a post-processing tool). turbo_lanczos.x performs a Lanczos recursion to obtain the $\beta, \gamma$, and $z$ coefficients, Eqs. (32), (35), and (36), while turbo_spectrum.x uses these coefficients to calculate the polarizability absorption spectra.

turboTDDFT has already been successfully applied to various systems, ranging from silicon nanoclusters [34,35], to photovoltaic systems [36-38]. In this section we describe the various components of the turboTDDFT software package. In the following we use the fullerene $C_{60}$ molecule as an example to illustrate the different steps for the computation and analysis of an optical absorption spectrum.

\subsection{Preliminary ground-state calculations}

In order to compute the optical spectrum of a system, a standard ground-state DFT calculation has to be performed first, yielding the KS orbital functions and energies for all the occupied levels. The information thus obtained is then used as input point for the linear response calculation. This ground-state computation is performed by the program pw.x, one of the key components of the QUANTUM ESPRESSO package. In Appendix A a sample input file for

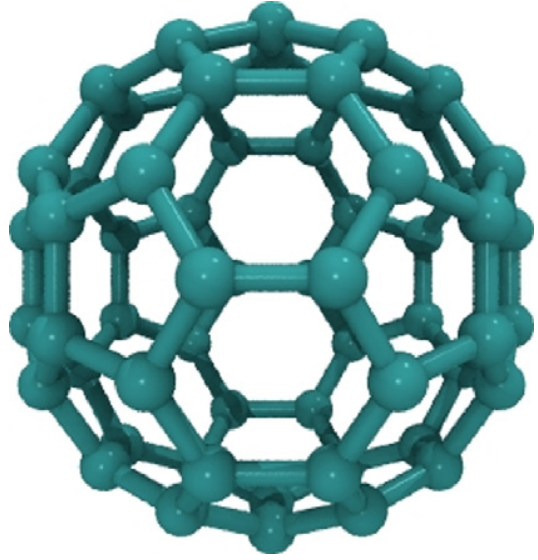

Fig. 3. Structure of the $C_{60}$ molecule.

pw. $x$ is reported for the case of the $C_{60}$ molecule (Fig. 3), using the PBE XC functional [41] and an ultrasoft (US) pseudopotential from the QUANTUM ESPRESSO database of potentials [22].

Two important limitations affect the present implementation of turboTDDFT: i) only integer occupations of KS states (the occupations keyword is set to 'fixed', which is the default); ii) only gamma-point $(\mathbf{k}=0)$ computations using realvalued wavefunctions are allowed. The K_POINTS input card must be set to Gamma. This is not a limitation for finite or extended disordered systems, whereas sampling the Brillouin zone of periodic systems would require the use of supercells. Work on implementing general k-point sampling and complex wavefunctions is in progress, but not included in the current release of turboTDDFT.

After successful completion of the ground-state computation, pw. $\mathrm{x}$ writes the KS orbitals and energies to disk, together with all relevant information about the system, like geometry, pseudopotentials, convergence parameters, etc. The TDDFT program turbo_lanczos.x reads all this data at start. It is therefore not necessary to re-define the system under study in the input file of turbo_lanczos.x.

\subsection{Lanczos recursions with turbo_Ianczos.x}

The Lanczos iterations performed by turbo_lanczos.x are by far the most time consuming step of the TDDFT computation. For the case of $C_{60}$, a sample input file for the turbo_lanczos. $x$ program is given in Appendix A. A list of all input variables of turbo_lanczos.x is given in Table B.1 of Appendix B. The 
(integer) input variable itermax set up the number of Lanczos iterations and so determines the dimension of the tridiagonal matrix Eq. (32); in general one can only check whether the number of iterations is sufficient to achieve an adequately converged representation at the post-processing stage, however, it is always possible to add more iterations to the calculation through using restart $=$.true. and increasing itermax. The strings defined in the input variables prefix and outdir identifies the system data on disk and must correspond to files created by pw. $\mathrm{x}$, easiest way to achieve this is to use the same settings in both of the programs.

The input variable ipol defines the direction of the polarization of the light $(i p o l=1,2,3$ for the Cartesian coordinates $\mathrm{x}, \mathrm{y}$, and $z$ ). For each direction of the polarization, one full Lanczos recursion chain needs to be computed. If the input variable ipol is set to the value 4, then turbo_lanczos.x will automatically perform three recursion chains, one for each direction; from this calculation it is possible to obtain the full polarizability tensor and not only the diagonal components.

After execution of turbo_lanczos.x, the computed $\beta$ and $\gamma$ coefficients are stored in the file prefix.beta_gamma_z.p, where $p=1-3$ for the three Cartesian directions. These portable ASCII text files are the only files required by turbo_spectrum. $x$ in the post-processing stage.

In Appendix A an example is provided on how to use turbo_ lanczos.x to compute the charge-density response. It is necessary to set charge_response=1 and in this case the card lr_post becomes mandatory. The variable omeg defines the value of the transition energy $\hbar \omega$ (in Ry) for which the density response is to be calculated. By setting the value of plot_type=1,2,3 a density output file can be obtained in xyzd, Xcrysden [44], or Gaussian cube formats, respectively.

\subsection{Obtaining the optical spectrum with turbo_spectrum.x}

From the computed recursion coefficients, the absorption spectrum can be obtained using Eq. (33). This task is performed by the program turbo_spectrum.x. The input variables for this program are documented in Table B.2 of Appendix B, and a sample input file for the case of the $C_{60}$ molecule is given in Appendix A.

The absorption spectrum is computed by solving the linear system of Eq. (37). In order to smooth out the spectrum near the eigenvalues of the tridiagonal matrix, this system is solved for complex frequencies, $\omega+i \varepsilon$. Setting $\varepsilon$ to a non-zero value (through the input variable epsil) amounts to convoluting the theoretical spectrum with a Lorentzian, or, alternatively, to broadening each individual spectral line. In the continuous region of the spectrum, where individual spectral lines are artifacts of the truncation of the Lanczos chain, the results thus obtained are independent on $\varepsilon$, whenever this parameter is smaller than the average distance between consecutive pseudo-discrete lines.

The convergence of the calculated spectrum in the desired energy range can be easily checked by varying the number of recursion coefficients used for the spectrum. This is set by the input variable itermax. It can take values up to the number of iterations which have been effectively performed previously using turbo_lanczos.x. If it turns out at this stage that not enough coefficients have been calculated before, it is possible to simply restart the turbo_lanczos.x code and to compute additional coefficients. This is possible without any loss of computer time, i.e. the code can be restarted from the last iteration.

As discussed in Section 3.2, the speed of convergence of the spectrum with respect to the number of Lanczos iterations can be drastically increased using extrapolated coefficients. Such extrapolations are used if the input variable terminator is set to osc or constant. In this case, the variable itermax is set to a high

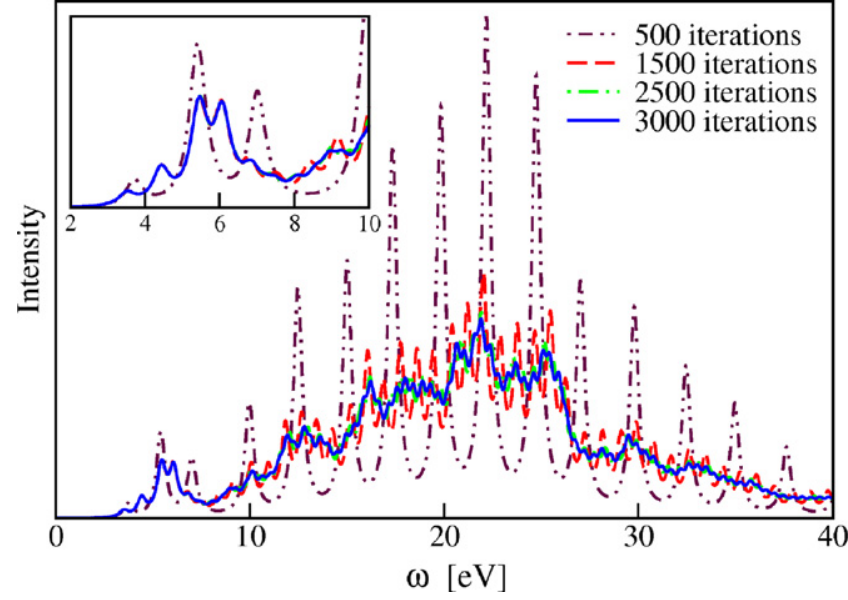

Fig. 4. Convergence the absorption spectrum of fullerene $C_{60}$ with respect to the number of Lanczos steps without extrapolation. An ultrasoft pseudopotential was used and a kinetic-energy cutoff of $30 \mathrm{Ry}$ to expand the wavefunctions. The comparison between the spectra at 2500 and 3000 iterations shows that 2500 iterations ensure a reasonably good convergence in a wide energy range. As highlighted in the inset, the low energy part of the spectrum converges faster and in this case 1500 iterations are enough for an accurate result.

value, e.g. 10,000, while the variable itermax 0 determines how many exactly computed coefficients are used. Extrapolated values for $\beta$ and $\gamma$ are used from itermax $0+1$ to itermax.

The post-processing code will generate a file prefix.plot. If in the previous turbo_lanczos.x calculation we have set up ipol=1,2,3, the prefix.plot will contain in the first column chi_p_p ( $p=i p o l)$, in the second the value of $\omega$, and in third and fourth columns the values of the real and imaginary part of the polarizability, Eq. (1), respectively. If in the previous turbo_lanczos.x calculation we have set up ipol=4, the post-processing code will compute the full polarizability tensor and the absorption coefficient. In the file prefix.plot the polarizability tensor components can be found in the lines starting with chi_p1_p2, where $\mathrm{p} 1$ and $\mathrm{p} 2$ can be 1,2,3, depending on the polarization direction. The absorption coefficient, computed as the product of the average of the trace of the polarizability with the frequency $\omega$, can be found in the lines starting with alpha.

In Fig. 4 we display the convergence of the spectrum of $C_{60}$ as a function of the number of Lanczos iterations. The technical details of this calculation are given in Appendix A. For this example 2500 iterations ensure a good convergence in a wide energy range, while in the low energy range (see inset) a smaller amount of Lanczos steps (about 1500) is enough to obtain accurate results. In Fig. 5 the extrapolation scheme of Section 3.2 is used to improve the convergence. By extrapolating the Lanczos chain, as few as 1200 iterations are enough to obtain a converged spectrum in a wide energy range.

The dependence of the Lanczos coefficients on the PW kineticenergy cutoff illustrated in Section 3.2 implies that simulations utilizing larger cutoffs usually require a larger number of iterations to converge. To illustrate this property an additional calculation using a norm-conserving (NC) pseudopotential, which requires a higher kinetic-energy cutoff of $70 \mathrm{Ry}$, has been performed. This example will also serve the purpose of comparing the performance of different functionals in TDDFT, namely the local density approximation PZ functional [42] and the generalized gradient approximation PBE [41] functional used in the previous example. As illustrated in Fig. 6(a), after 3000 iterations the spectrum still exhibits wide oscillations, while in Fig. 4 the same number of iterations was providing converged results. Also in this case the extrapolation scheme of Section 3.2 can be used to sensibly reduce the 


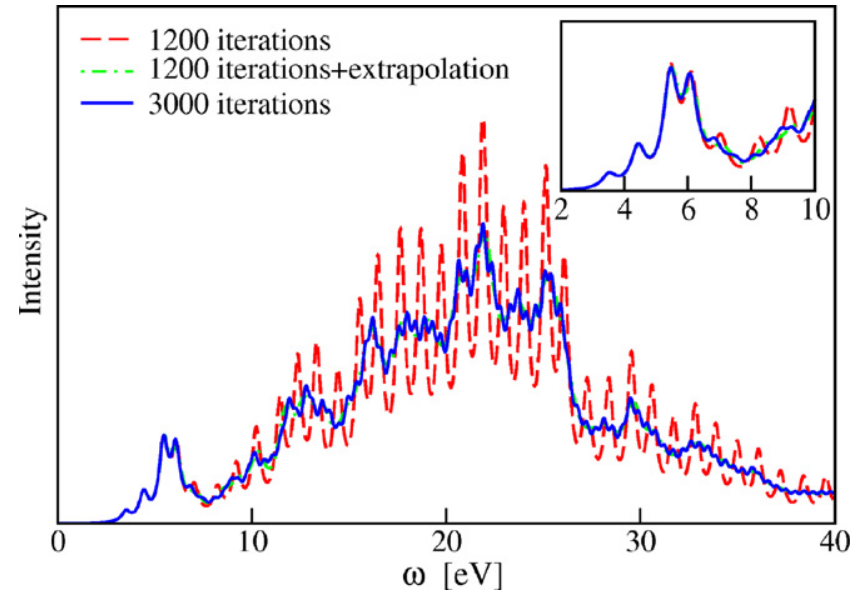

Fig. 5. Improvement in the convergence of the absorption spectrum of fullerene $C_{60}$ using the extrapolation technique described in Section 3.2. The spectrum computed at 1200 iterations still shows wide oscillations if compared to the converged result at 3000 iterations. If after 1200 iterations the Lanczos coefficients are extrapolated to a sufficiently large number $(10,000$ in this case), the computed spectrum becomes almost indistinguishable from the converged result.

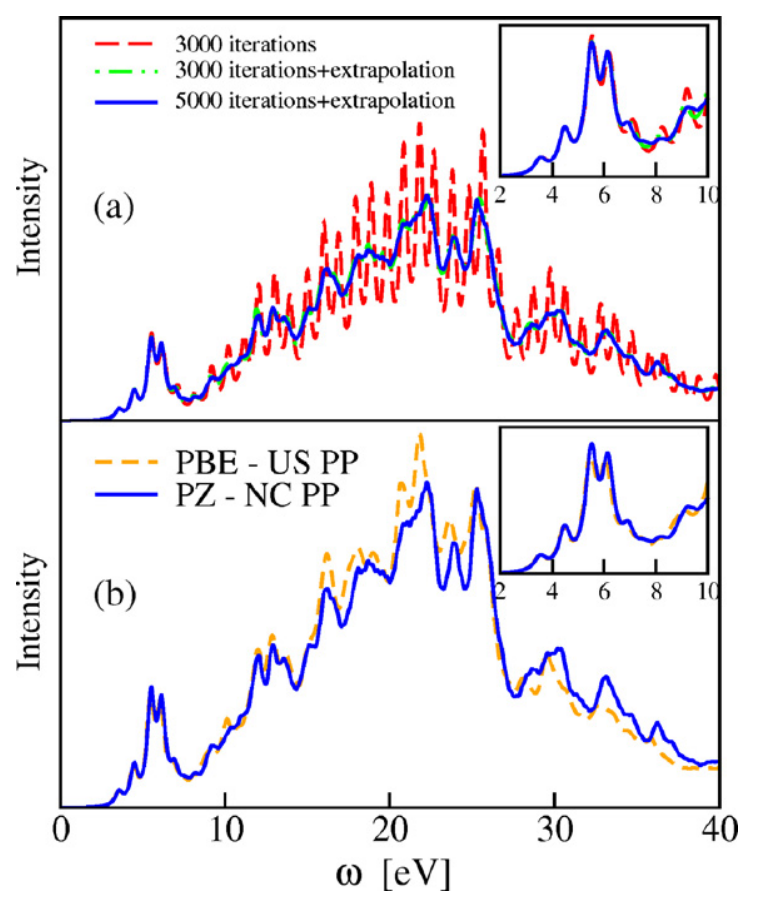

Fig. 6. (a): Convergence properties of the spectrum of fullerene using normconserving pseudopotentials. The larger cutoff ( $70 \mathrm{Ry}$ ) used in this calculation requires a larger number of iterations compared to the ultrasoft pseudopotential calculation presented in Fig. 4. Indeed, after 3000 iterations the spectrum still shows very large oscillations and only using the Lanczos extrapolation scheme it is possible to converge the results. (b): Comparison of the spectrum of fullerene obtained using the PBE functional and ultrasoft pseudopotentials (PBE-US) with the spectrum obtained using the PZ functional and norm-conserving pseudopotentials (PZ-NC). Despite the different computational details the two spectra are in quite good agreement.

computational workload. In Fig. 6(b) the spectra obtained using different pseudopotentials (US and NC) and functionals (PBE and $\mathrm{PZ}$ ) are compared. The agreement is excellent in the low-energy part of the spectrum. At higher energy the structure and intensity become slightly different. This is only a minor issue since, as illustrated in Ref. [10], the fine structure (but not the overall

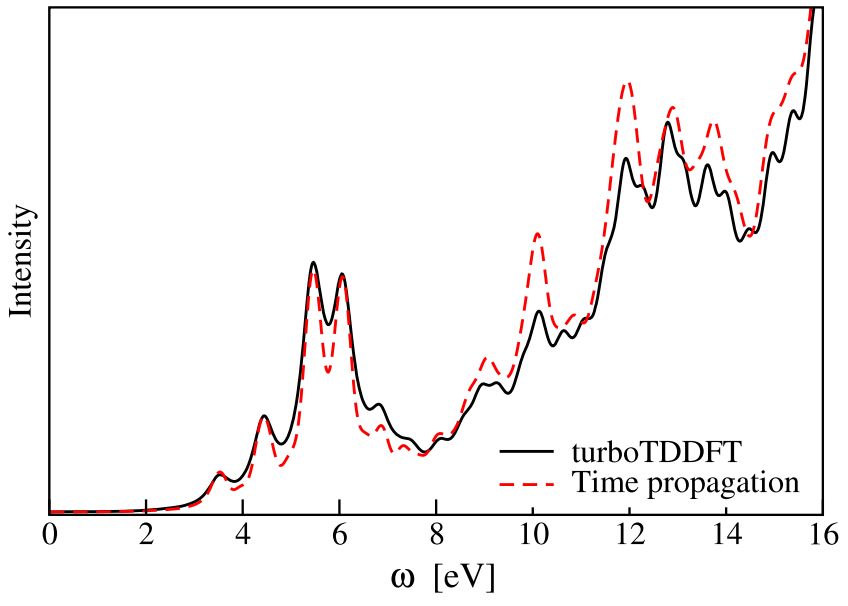

Fig. 7. Comparison of the absorption spectrum of fullerene $C_{60}$ computed with the Liouville-Lanczos method described in this work and with a time propagation algorithm. Both calculations are performed starting from the same ground state.

shape) of the continuum is a spurious effect of the finite supercell size.

\section{Installation instructions}

turboTDDFT is distributed as a source code, and needs compiling for the target environment. The installation procedure is tightly bound with the installation of QUANTUM ESPRESSO, and it is not any different than compiling any other post-processing code contained therein. QUANTUM ESPRESSO and turboTDDFT makes use of the GNU autotools. After extracting in the root directory of the QUANTUM ESPRESSO tree, issuing the two UNIX commands

. /configure make tddfpt

will create the turboTDDFT executables in the bin/ folder of the QUANTUM ESPRESSO tree. Further detailed installation instructions can be found in the documentation that comes with the QUANTUM ESPRESSO distribution.

\section{Comparison with time propagation and sum rules}

We have also compared the absorption spectrum of C60, calculated using turboTDDFT with the spectrum obtained from an explicit time propagation of the KS equations $[10,39,40]$. For the time propagation we employed the same cell, basis set, functional, and pseudopotential given in Appendix A. Real-time propagation are performed using a fourth-order polynomial expansion of the propagator $\exp (-i H(t) \Delta t / \hbar)$, together with the so-called enforced time-reversal symmetry (ETRS) method [43]. A time step $\Delta t$ of $9.67 \cdot 10^{-19} \mathrm{~s}$ is employed, for a total simulation time of $13.4 \mathrm{fs}$. The absorption spectrum thus obtained agrees well with the spectrum obtained by applying the Lanczos recursions, as shown in Fig. 7. The good agreement of the two spectra, computed in one case using linear-response algorithms and in the other case a general time propagation, further validates our implementation of TDDFPT.

Optical susceptibilities satisfy many sum rules, the most fundamental of which is probably the Thomas-Reiche-Kuhn [25-27] 
(or $f$-sum) rule, which relates the integral of the absorption coefficient of a molecular system to the number of electrons contained in it:

$$
\begin{aligned}
f & =\operatorname{Im} \sum_{j} \int_{0}^{\infty} \alpha_{j j}(\omega) \omega d \omega \\
& =\frac{3}{2} N_{e} \pi,
\end{aligned}
$$

$N_{e}$ being the number of electrons (of valence electrons, in a pseudopotential calculation) in the system. The Liouville-Lanczos approach to TDDFT can be demonstrated to satisfy the f-sum rule exactly, for any number of Lanczos iterations [45]. This is only true when utilizing local pseudopotentials. When non-local NC or US pseudopotentials are used, violations of the f-sum rule are to be expected. In the two calculations for $C_{60}$ presented above, the f-sum violation is extremely small when using NC pseudopotentials $(\Delta f / f \approx 0.007)$, whereas it is one order of magnitude larger $(\Delta f / f \approx 0.078)$, when utilizing US pseudopotentials.

Let us finally comment on typical CPU times in the example of the C60 molecule. On an IBM SP6 machine, employing 32 processors, the time propagation (13900 steps) took in total 48.7 hours ( 0.21 minutes per time step). On the same machine and for the same system, turboTDDFT employed 1.8 hours (0.044 minutes per Lanczos step, for a total of 2500 steps). Even though the precise CPU requirements for time propagations depend heavily on the implementation and employed basis sets, this comparison shows clearly how performant turboTDDFT can be.

\section{Conclusions}

In this work we have presented the turboTDDFT code, that implements the Liouville-Lanczos approach to time-dependent density-functional perturbation theory. A detailed description of the use of the code has been provided considering the example of fullerene $\mathrm{C}_{60}$

The turboTDDFT implementation has a numerical scalability comparable to ground state DFT calculations and does not require the explicit calculation of any empty electronic state. Furthermore, the use of the Lanczos algorithm allows computing of optical spectra in a very wide energy range and easy verification of the $f$-sum rule.

In the current implementation hybrid functionals are not available and the calculations are restricted to a single $\mathrm{k}$ point (gamma). These features will be included in future releases.

In the same spirit as the QUANTUM ESPRESSO project, turboTDDFT provides scientists worldwide a well commented and open-source framework for implementing their ideas. It is in our best hopes that turboTDDFT can benefit from the already well established user community of QUANTUM ESPRESSO for incorporating new ideas and keep growing in the future. turboTDDFT is hosted in a community accessible SVN repository and hence, apart from releases in QUANTUM ESPRESSO, those who are willing to test the latest experimental implementations are welcome to do so and contribute with their feedback.

\section{Acknowledgements}

R.G. thanks the DEISA Consortium (www.deisa.eu), co-funded through the EU FP6 project RI-031513 and the FP7 project RI222919, for support within the DEISA Extreme Computing Initiative. S.B. gratefully acknowledges financial support from MIUR through PRIN2008.

\section{Appendix A. Sample input files}
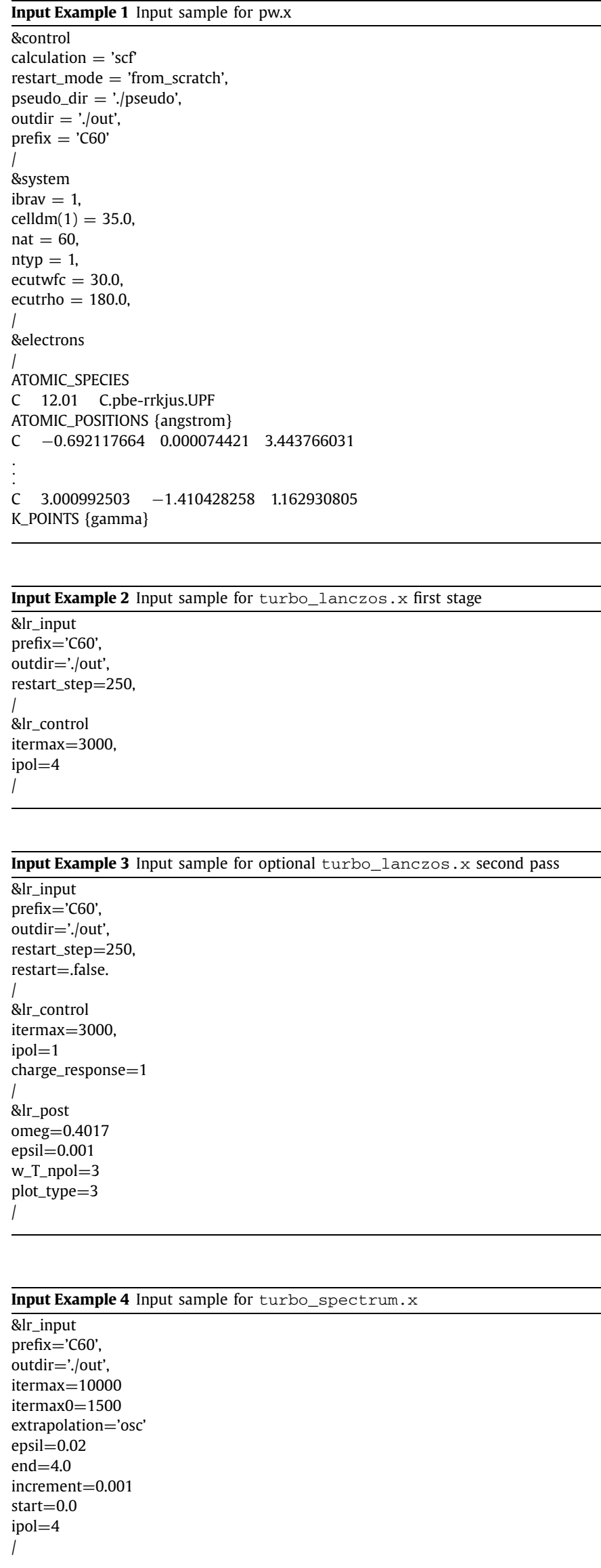
Appendix B. Input variables

Table B.1

Input variables for turbo_lanczos.x.

\begin{tabular}{|c|c|c|c|}
\hline Card & Variable name & Default value & Description \\
\hline \multirow[t]{6}{*}{ lr_input } & prefix & 'pwscf' & $\begin{array}{l}\text { Sets the prefix for generated and read files. The files generated by the ground state } \\
\text { pw. } x \text { run should have this same prefix. }\end{array}$ \\
\hline & outdir & '.l' & $\begin{array}{l}\text { The directory that contains the run critical files, which include the files generated } \\
\text { by ground state pw.x run. }\end{array}$ \\
\hline & wfedir & unset & $\begin{array}{l}\text { The directory where the scratch files will be written and read. Restart related files } \\
\text { are always written to outdir. }\end{array}$ \\
\hline & restart & false. & $\begin{array}{l}\text { When set to .true., turbo_lanczos.x will attempt to restart from a previous } \\
\text { interrupted calculation (see restart_step variable). }\end{array}$ \\
\hline & restart_step & itermax & $\begin{array}{l}\text { The code writes restart files every restart_step iterations. Restart files are } \\
\text { automatically written at the end of itermax Lanczos steps. }\end{array}$ \\
\hline & lr_verbosity & 1 & This integer variable controls the amount of information written in standard output. \\
\hline \multirow[t]{6}{*}{ lr_control } & itermax & 500 & Number of iterations to be performed. \\
\hline & ipol & 1 & $\begin{array}{l}\text { An integer variable that determines which element of the dynamical polarizability } \\
\text { will be computed: } 1 \rightarrow \alpha_{x x}(\omega), 2 \rightarrow \alpha_{y y}(\omega) \text {, and } 3 \rightarrow \alpha_{z z}(\omega) \text {. When set to } 4 \text {, three } \\
\text { Lanczos chains are sequentially performed and the full polarizability tensor and the } \\
\text { absorption coefficient are computed. }\end{array}$ \\
\hline & nipol & 1 if ipol $<4$; 3 if ipol $=4$ & $\begin{array}{l}\text { Determines the number of zeta coefficients to be calculated for a given polarization } \\
\text { direction. }\end{array}$ \\
\hline & 1 tammd & false. & $\begin{array}{l}\text { When set to .true. the Tamm-Dancoff approximation is used in constructing the } \\
\text { Liouvillian. }\end{array}$ \\
\hline & no_hxc & false. & $\begin{array}{l}\text { When set to true. the change in the internal field (Hartree and exchange-correlation) } \\
\text { is ignored in the calculation, resulting in an independent electron approximation. }\end{array}$ \\
\hline & charge_response & 0 & $\begin{array}{l}\text { When set to } 1 \text {, the code computes the response of the charge density and writes it } \\
\text { into a file format determined by the variable plot_type. Setting } \\
\text { charge_response to } 1 \text { makes the presence of the card lr_post mandatory. }\end{array}$ \\
\hline \multirow[t]{5}{*}{ lr_post } & omeg & 0.0 & $\begin{array}{l}\text { The response of the charge density is calculated for this transition energy (in } \\
\text { Rydberg units) }\end{array}$ \\
\hline & epsil & 0.0 & The broadening/damping term (in Rydberg units). \\
\hline & beta_gamma_z_prefix & 'pwscf' & $\begin{array}{l}\text { The prefix of the file where the beta gamma zeta coefficients from the first } \\
\text { calculation can be set manually using this parameter. The file outdir/beta_ } \\
\text { gamma_z_prefix.beta_gamma_z.x (where } x=1-3 \text { ) must exist. }\end{array}$ \\
\hline & w_T_npol & 1 & $\begin{array}{l}\text { Number of polarization directions considered in the previous calculation. It must be } \\
\text { set to } 3 \text { if in the previous calculation } i p o l=4 \text {, it must be set to } 1 \text { otherwise. }\end{array}$ \\
\hline & plot_type & 1 & $\begin{array}{l}\text { An integer variable that determines the format of the file containing the } \\
\text { charge-density response. } 1 \text { : A file containing the } \mathrm{x} \text { y } \mathrm{z} \text { grid coordinates and the } \\
\text { corresponding value of the density is produced } 2 \text { : The density response is written in } \\
\text { Xcrysden format } 3 \text { : The density response is written in the Gaussian cube format. }\end{array}$ \\
\hline
\end{tabular}

Table B.2

Input variables for turbo_spectrum. $x$.

\begin{tabular}{|c|c|c|c|}
\hline Card & Variable name & Default value & Description \\
\hline \multirow[t]{12}{*}{ lr_input } & prefix & 'pwscf & Prefix of the files generated by the previous turbo_lanczos. $x$ run. \\
\hline & outdir & $\therefore$ & $\begin{array}{l}\text { The directory where the output files produced by the previous turbo_lanczos.x } \\
\text { run are stored. }\end{array}$ \\
\hline & itermax0 & 500 & Number of Lanczos coefficients to be read from the file. \\
\hline & itermax & 500 & $\begin{array}{l}\text { The total number of Lanczos coefficients that will be considered in the calculation of } \\
\text { the polarizability/absorption coefficient. If itermax }>\text { itermax } 0 \text {, the Lanczos } \\
\text { coefficients in between itermax } 0+1 \text { and itermax will be extrapolated. }\end{array}$ \\
\hline & extrapolation & 'no' & $\begin{array}{l}\text { Sets the extrapolation scheme. 'osc' = bi-constant extrapolation; 'constant' }= \\
\text { constant extrapolation; 'no' = no extrapolation. }\end{array}$ \\
\hline & epsil & 0.02 & The broadening/damping term (in Rydberg units). \\
\hline & units & 0 & Unit system used. 0 : Rydbergs; 1 : Electron volts 2: Nanometers/Electron volts. \\
\hline & start & 0.0 & $\begin{array}{l}\text { The polarizability and the absorption coefficient are computed starting from this } \\
\text { value. In units set by the units variable. }\end{array}$ \\
\hline & end & 2.5 & $\begin{array}{l}\text { The polarizability and the absorption coefficient are computed up to this value. In } \\
\text { units set by the units variable. }\end{array}$ \\
\hline & increment & 0.001 & $\begin{array}{l}\text { Incremental step used to define the mesh between start and end. In units set by } \\
\text { the units variable. }\end{array}$ \\
\hline & ipol & 1 & $\begin{array}{l}\text { An integer variable that determines which element of the dynamical polarizability } \\
\text { will be computed: } 1 \rightarrow \alpha_{x x}(\omega), 2 \rightarrow \alpha_{y y}(\omega) \text {, and } 3 \rightarrow \alpha_{z z}(\omega) \text {. When set to } 4 \text { the } \\
\text { polarizability tensor and oscillator strength function are computed. }\end{array}$ \\
\hline & verbosity & 0 & This integer variable Controls the output verbosity. \\
\hline
\end{tabular}




\section{References}

[1] P. Hohenberg, W. Kohn, Phys. Rev. 136 (1964) B864.

[2] W. Kohn, L.J. Sham, Phys. Rev. 140 (1965) A1133.

[3] E. Runge, E.K.U. Gross, Phys. Rev. Lett. 52 (1984) 997.

[4] M.A.L. Marques, C.A. Ullrich, F. Nogueira, A. Rubio, K. Burke, E.K.U. Gross (Eds.) Time Dependent Density Functional Theory, Springer-Verlag, Berlin, Heidelberg, 2006.

[5] A. Dreuw, J.L. Weisman, M. Head-Gordon, J. Chem. Phys. 119 (2003) 2943.

[6] D. Rocca, D. Lu, G. Galli, J. Chem. Phys. 133 (2010) 164109.

[7] P. Ghosh, R. Gebauer, J. Chem. Phys. 132 (2010) 104102.

[8] B. Walker, A.M. Saitta, R. Gebauer, S. Baroni, Phys. Rev. Lett. 96 (2006) 113001.

[9] D. Rocca, Y. Saad, R. Gebauer, S. Baroni, J. Chem. Phys. 128 (2008) 154105.

[10] B. Walker, R. Gebauer, J. Chem. Phys. 127 (2007) 164106.

[11] D. Rocca, Time-dependent density-functional perturbation theory: new algorithms with applications to molecular spectra, SISSA PhD thesis, http:// www.sissa.it/cm/thesis/2007/Dario_Rocca_PhD_Thesis.pdf.

[12] E.K.U. Gross, W. Kohn, Phys. Rev. Lett. 55 (1985) 2850.

[13] M. Petersilka, U.J. Gossmann, E.K.U. Gross, Phys. Rev. Lett. 76 (1996) 1212.

[14] M.E. Casida, in: D.P. Chong (Ed.), Recent Advances in Density Functional Methods, Part I, World Scientific, Singapore, 1995, p. 155.

[15] A. Castro, H. Appel, Micael Oliveira, C.A. Rozzi, X. Andrade, F. Lorenzen, M.A.L. Marques, E.K.U. Gross, A. Rubio, Phys. Stat. Sol. B 243 (2006) 2465.

[16] M.A.L. Marques, A. Castro, G.F. Bertsch, A. Rubio, Comput. Phys. Commun. 151 (2003) 60.

[17] S. Baroni, P. Giannozzi, A. Testa, Phys. Rev. Lett. 58 (1987) 1861.

[18] S. Baroni, S. de Gironcoli, A. Dal Corso, P. Giannozzi, Rev. Mod. Phys. 73 (2001) 515.

[19] http://www.gnu.org/licenses/gpl.html.

[20] S. Scandolo, P. Giannozzi, C. Cavazzoni, S. de Gironcoli, A. Pasquarello, S. Baroni, Z. Kristallog. 220 (2005) 574.

[21] P. Giannozzi, et al., J. Phys. Cond. Mat. 21 (2009) 395502.

[22] http://www.quantum-espresso.org.
23] F. Bassani, M. Altarelli, Interaction of radiation with condensed matter, in Handbook of Synchroton Radiation, North Holland, Amsterdam, 1983, pp. 465597.

[24] R. Bauernschmitt, R. Ahlrichs, Chem. Phys. Lett. 256 (1996) 454

[25] W. Thomas, Naturwissenschaften 13 (1925) 627.

[26] W. Kuhn, Z. Phys. 33 (1925) 408.

[27] F. Reiche, W. Thomas, Z. Phys. 34 (1925) 510.

[28] A. Baldereschi, E. Tosatti, Phys. Rev. B 17 (1978) 4710

[29] S. Baroni, R. Resta, Phys. Rev. B 33 (1986) 7017.

[30] J. Tobik, A. Dal Corso, J. Chem. Phys. 120 (2004) 9934

[31] D.W. Bullet, R. Haydock, V. Heine, M. Kelly, Solid State Physics, vol. 35, Academic, New York, 1980.

[32] Y. Saad, SIAM J. Numer. Anal. 19 (1982) 485.

[33] S. Baroni, R. Gebauer, O.B. Malcioglu, et al., J. Phys. Cond. Mat. 22 (2010) 074204

[34] B.G. Walker, S.C. Hendy, R. Gebauer, R.D. Tilley, Eur. Phys. J. B 66 (2008) 7.

[35] A. Gali, M. Vörös, D. Rocca, G.T. Zimanyi, G. Galli, Nano Lett. 9 (2009) 3780.

[36] D. Rocca, R. Gebauer, F. De Angelis, M.K. Nazeeruddin, S. Baroni, Chem. Phys. Lett. 475 (2009) 49.

[37] L. Huang, D. Rocca, S. Baroni, K.E. Gubbins, M. Buongiorno Nardelli, J. Chem Phys. 130 (2009) 194701

[38] F. De Angelis, S. Fantacci, R. Gebauer, J. Phys. Chem. Lett. 2 (2011) 813.

[39] R. Martin, Electronic Structure: Basic Theory and Practical Methods, Cambridge University Press, Cambridge, UK, 2004 (Chapter 20.4).

[40] K. Yabana, G.F. Bertsch, Int. J. Quant. Chem. 75 (1999) 55.

[41] J.P. Perdew, K. Burke, M. Ernzerhof, Phys. Rev. Lett. 77 (1996) 3865.

[42] J.P. Perdew, A. Zunger, Phys. Rev. Lett. 23 (1981) 5048.

[43] A. Castro, M.A.L. Marques, A. Rubio, J. Chem. Phys. 121 (2004) 3425.

[44] A. Kokalj, Comp. Mater. Sci. 28 (2003) 155, Code available from http:/ www.xcrysden.org/.

[45] S. Baroni, R. Gebauer, in: M.A.L. Marques, N. Maitra, F. Nogueira, E.K.U. Gross, A. Rubio (Eds.), Fundamentals of Time-Dependent Density Functional Theory, Springer, Berlin, in preparation. 